\title{
Correspondence
}

Editor: Ian Pullen

Contents: Personality and outcome of depression/ Violence in Alzheimer's disease/Oestrogen therapy and the menopause/The 'new cross-cultural psychiatry'/Ethnic minorities and the psychiatric system/Benzodiazepine withdrawal/Limitations of double-blind trials/The Lomax affair/Education for the 21 st century/British Neuropsychiatry Association/ The Bowdlerisation of psychiatry/Social order/ mental disorder/Treatment of depression with pumpkin seeds/Down's syndrome, dementia and myoclonic jerks/How informed and binding is informed consent.

\section{Personality and outcome of depression}

SIR: Andrews et al (Journal, July 1990, 157, 13-18) present a very interesting study of personality and long-term outcome in depression, but there are alternative explanations to the data presented.

The authors follow the tradition of regarding endogenous and neurotic depression as a dichotomy. Their study shows the negative influence of personality on the outcome of neurotic depression (ND) but not endogenous depression (ED). Andreasen has indicated factors which support a dichotomy view (Andreasen, 1980). The findings by Professor Andrews et al exemplify one of these.

The paper also suggests that endogenous and neurotic depression have different courses with 'episodic' or 'more insidious' features respectively. The data presented is open to different interpretation. A total of $57 \%$ of their ON group (other neurosis presenting as depression) have drifted into ND by the end of the study. This suggests a high degree of overlap between the two groups. It is therefore just as reasonable to sum the two groups together as to make a possibly artificial division between them. Doing this the combined ND+ON group may then be compared with the ED group. In the combined group $(n=127)$, $16 \%$ remained well, $71 \%$ suffered a recurrence of some degree and $18 \%$ were incapacitated during the follow-up period. In the ED group $(n=66)$ these figures are $15 \%, 60 \%$, and $16 \%$ respectively. These findings seem more remarkable for their similarities than their differences. From a clinical perspective both ED and ND appear to have similar courses.
The authors also suggest that the characteristics of ND more closely resemble ON than ED. In view of the diagnostic drift from ON to ND this is perhaps not surprising.

In the view of the Newcastle group which Professor Andrews et al favour, a personality inadequacy is one factor defining neurotic rather than endogenous depression (Carney et al, 1965). This study demonstrates that in depression, where personality factors are initially an aetiological factor, they remain a factor influencing the future recurrence of illness. Where personality factors are less important, in an initial endogenous diagnosis, they remain less of an influence in the outcome of the illness.

Overall, the courses of ND and ED appear only a little different in outcome. What differences there are can be explained by the modifying effects of personality. The case for a dichotomy is not proven.

Mapperley Hospital

A. J. AsPIN

Porchester Road

Nottingham NG3 6AA

\section{References}

Andreasen, N. C. (1980) Concepts, diagnosis and classification. In Handbook of Affective Disorders (ed. E. Paykel), pp. 22-44. Edinburgh: Churchill Livingstone.

CARNEY, M. W. P., Roth, M. \& GARSide, R. F. (1965) The diagnosis of depressive syndromes and the prediction of ECT responses. British Journal of Psychiatry, 3, 659-674.

AUTHORS' REPLY: We have published two papers on the long-term outcome of our cohort of depressive patients (Journal, June 1988, 153, 752-757 and Journal, July 1990, 157, 13-18). Your correspondent appears to have overlooked the former paper in which we report that the morbidity at 15 years was the same for patients with endogenous or neurotic depression. We thank your correspondent for restating what we have already published and indeed stated again in the abstract of the second paper: "diagnosis at index admission did not predict overall outcome" (1990, p.13). We noted that despite the similarity in outcome, "patients with endogenous depression, an apparently stable diagnosis, had longer index admissions, were readmitted sooner, but 